\title{
Sculpting the spin-wave response of artificial spin ice via microstate selection
}

\author{
D. M. Arroo $\odot,{ }^{1,2, *}$ J. C. Gartside, ${ }^{1}$ and W. R. Branford ${ }^{1, \dagger}$ \\ ${ }^{1}$ Blackett Laboratory, Imperial College, Prince Consort Road, London SW7 2AZ, England, United Kingdom \\ ${ }^{2}$ London Centre for Nanotechnology and Department of Physics and Astronomy, University College London, 17-19 Gordon Street, London \\ WC1H OAH, England, United Kingdom
}

(Received 4 May 2018; revised manuscript received 6 December 2019; published 23 December 2019)

\begin{abstract}
Artificial spin ice (ASI) systems have emerged as promising hosts for magnonic applications due to a correspondence between their magnetic configuration and spin dynamics. Though it has been demonstrated that spin-wave spectra are influenced by the ASI microstate the precise nature of this relationship has remained unclear. Recent advances in controlling the magnetic configuration of ASI make harnessing the interplay between spin dynamics and the microstate achievable. This could allow diverse applications including reconfigurable magnonic crystals and programmable microwave filters. However, extracting any novel functionality requires a full understanding of the underlying spin-wave/microstate interaction. Here, we present a systematic analysis of how the microstate of a honeycomb ASI system affects its spin-wave spectrum through micromagnetic simulations. We find the spectrum to be highly tunable via the magnetic microstate, allowing the (de)activation of spin-wave modes and band-gap tuning via magnetic reversal of individual nanoislands. Symmetries of ASI systems and the chirality of "monopole" defects are found to play important roles in determining the high-frequency magnetic response.
\end{abstract}

DOI: 10.1103/PhysRevB.100.214425

\section{INTRODUCTION}

Artificial spin ice (ASI) systems consist of arrays of nanoscale ferromagnetic islands the frustrated interactions of which lead to vastly degenerate low-energy states [1-3]. For ASI systems on a honeycomb lattice, such energy states are well characterized by the number of vertices at which islands have their magnetizations pointing either all in or all out, known as "monopole defects" [4-6]. The ground-state manifold consists of all magnetic configurations of the islands ("microstates") with no monopole defect at any vertex [7].

Recently, ASI systems have emerged [8,9] as the potential basis of reconfigurable magnonic devices [10-12] in which the high-frequency magnetic response may be altered by varying the system microstate, leading to a reprogrammable magnonic band structure [13]. The large degeneracy of the ASI ground state could then be used to overcome the limited range of easily accessible states available in previously explored reconfigurable magnonic crystals $[14,15]$ as well as providing enhanced stability to fluctuations. Working in the other direction, measuring high-frequency eigenmode spectra could be employed as a way of "reading out" ASI microstates in data processing applications.

A correspondence between the high-frequency dynamics of ASI systems and their microstate would thus be beneficial for a range of applications. Though a link between microstates and high-frequency dynamics has been demonstrated for some ASI systems [16], so far various aspects of this link remain poorly understood. In particular it is not clear from previous

\footnotetext{
*d.arroo@ucl.ac.uk

†w.branford@imperial.ac.uk
}

work under what conditions different microstates have unique spin-wave spectra.

The majority of experimental studies on the highfrequency behavior of ASI systems to date have involved measuring ferromagnetic resonance (FMR) in large arrays subject to an external swept field $[9,17-21]$. Since the precise magnetic microstate during such sweeps is unknown, it is unclear whether the observed changes in resonant frequencies depend only on the number of islands that have been reversed or whether the precise magnetic configuration of the array plays a role. Moreover, while the large number of magnetic microstates available in ASI systems has been posited as the source of a corresponding large number of high-frequency behaviors [17], the number of distinct regimes observed in swept-field FMR experiments so far has been modest. This may be explained by return-point memory effects in ASI systems [22] leading to only a small subset of the possible microstates being explored during a field sweep, but it remains to be established how many of the exponentially large number of microstates possess unique signatures in the high-frequency response.

More recent work $[23,24]$ has focused on isolated ASI vertices consisting of three islands at $120^{\circ}$ to one another. These studies have clarified the contribution of individual vertices to the overall high-frequency response and highlighted how deviations from uniform magnetization in islands can lead to mode softening, but these results also rely on swept fields and since they concern individual vertices they cannot explore the rich microstate space and long-range interactions of extended ASI arrays.

Here, the link between the microstate of an ASI system and its high-frequency dynamics is analyzed in detail via micromagnetic simulations [25], with a view to isolating the 


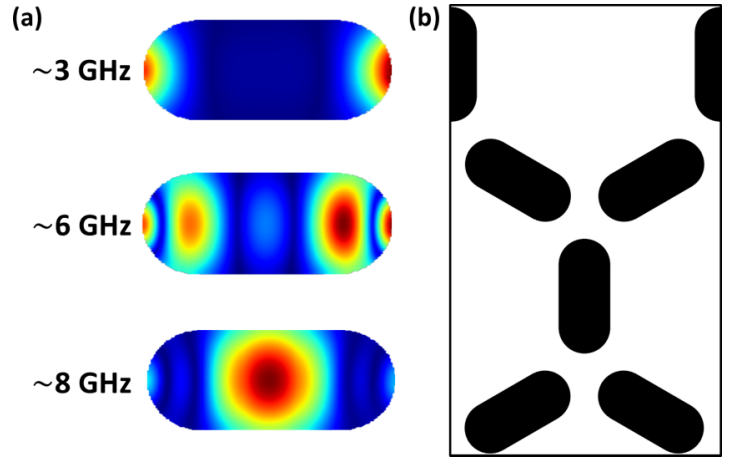

FIG. 1. (a) Power distributions for the three eigenmodes observed for an isolated ferromagnetic island. The mode around $8 \mathrm{GHz}$ is localized in the bulk of the island and has the largest amplitude, while the two lower-frequency modes are localized at the edges. (b) A six-island artificial spin ice unit cell simulated with periodic boundary conditions, allowing $2^{6}$ configurations with up to four monopole defects per unit cell to be explored.

role of the ASI microstate in the spin-wave response from effects of external magnetic fields. The results are relevant to assessing how ASI systems may be incorporated into the ever growing range of reconfigurable magnonic device concepts for low-power information processing [10].

\section{SIMULATION METHODOLOGY}

Spin-wave eigenmode spectra were generated for honeycomb ASI systems with MUMAX3 [26] by applying an out-ofplane field pulse to and sampling the evolution of the system's magnetization every $20 \mathrm{ps}$ over the subsequent $5 \mathrm{~ns}$. The pulse applied was uniform in space (so that excited modes correspond to $\vec{k}=0$ uniform precession modes) and varied as a sinc function in time with a maximum amplitude of $20 \mathrm{mT}$, so that frequencies up to $25 \mathrm{GHz}$ were excited equally. By applying a Fourier transform to the magnetization of each micromagnetic cell, the intensity of precession can then be mapped out as a function of both frequency and position [27,28].

Simulations were performed for $130 \times 290 \times 20$-nm islands with micromagnetic parameters matching those of permalloy, with $M_{\mathrm{S}}=800 \mathrm{kA} / \mathrm{m}, A_{\mathrm{ex}}=13 \mathrm{pJ} / \mathrm{m}$, and $\alpha=$ 0.006 . The basic form of the eigenmode spectra was found to derive from that of the single-island spectrum, exhibiting three modes: Two localized at the island edges and one in the bulk [Fig. 1(a)]. In an ASI array, magnetostatic interactions caused these modes to shift in frequency and split into multiplets.

To gain an overview of the effect of the magnetic configuration on the high-frequency dynamics of an extended honeycomb ASI array, the system was reduced to a plaquette of six islands with four vertices [Fig. 1(b)] and simulated with periodic boundary conditions. Because the magnetization of the ferromagnetic islands in ASI systems is strongly constrained by shape anisotropy to lie along the island axis, the islands are commonly treated as macrospins [2], giving a total of $2^{6}=64$ configurations for a six-island system. Spectra were generated at remanence for the entire configuration space, allowing the effect on the eigenmode spectrum of a

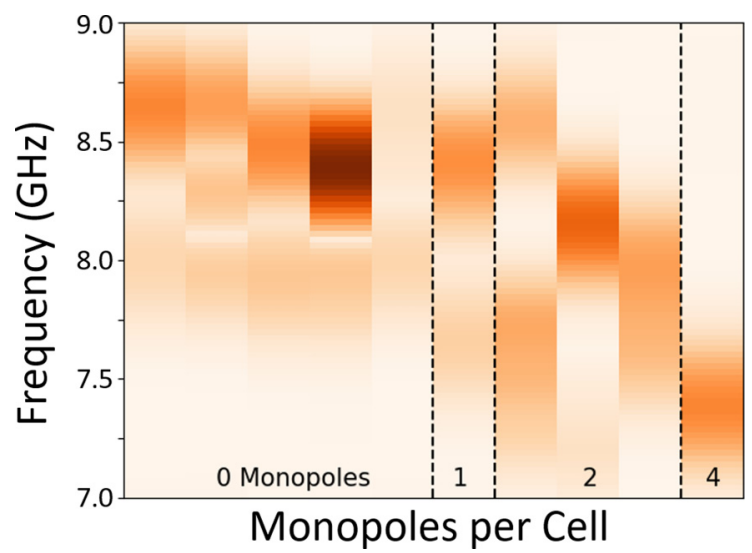

FIG. 2. Spectra around the $8-\mathrm{GHz}$ bulk mode for the ten microstates that are distinguishable in the macrospin picture for the six-island unit cell shown in Fig. 1(b), arranged in order of monopole density. The remaining 54 available microstates may be mapped onto these ten microstates via rotations and global spin reversals, and reproduce their corresponding spectra.

range of different configurations and monopole densities to be probed.

\section{RESULTS AND DISCUSSION}

Within a macrospin picture, the number of microstates with distinguishable mode spectra is significantly reduced by the fact that many configurations may be mapped onto one another via global spin reversals and rotations. The dynamics of ferromagnetic systems is described by the Landau-Lifshitz equation

$$
\frac{\partial \mathbf{M}}{\partial t}=\gamma \mu_{0} \mathbf{M} \times \mathbf{H}_{\mathrm{eff}}
$$

which has no explicit dependence on direction and is invariant under time reversal (i.e., a global spin reversal), so that microstates separated only by these transformations have identical spectra. The time-reversal symmetry is in fact broken when damping is included, but in a steady state the damping is precisely canceled out by the driving ac field so that it can be neglected.

For a finite honeycomb system with $n$ macrospins and full hexagonal symmetry the number of spectrally distinguishable microstates is thus reduced from $2^{n}$ to $2^{n / 2}$, putting a limit on the versatility of ASI systems as reconfigurable magnonic components. Here a reduced six-island system [Fig. 1(b)] is studied with periodic boundary conditions, so that translational symmetry and the broken hexagonal symmetry of the rectangular plaquette that was used leave ten configurations with distinct spectra. These ten configurations may be meaningfully sorted by the number of monopole defects per cell, since the total monopole number is invariant under both rotations and global spin reversals.

The ASI spectra generated showed bands corresponding to the three modes observed in an isolated ferromagnetic island (the band corresponding to the bulk mode around $8 \mathrm{GHz}$ is shown in Fig. 2), with interisland interactions causing peaks to shift in frequency or split into multiple peaks depending on the 

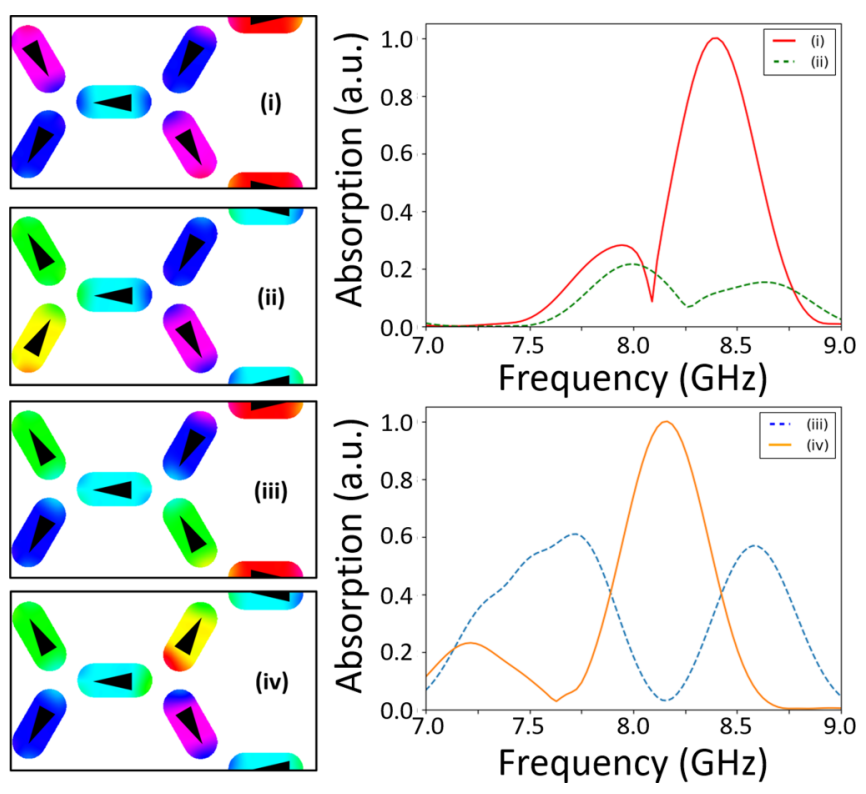

FIG. 3. Moving between two zero-monopole configurations (i, ii) or two two-monopole configurations (iii, iv) leads to significant enhancement/suppression of the 8-GHz bulk mode, suggesting applications as "ON" and "OFF" states. The enhancement factor depends on the strength of the interisland coupling.

microstate. It was found that for the bulk mode around $8 \mathrm{GHz}$ the macrospin picture indeed accounts for ten distinct spectra, whereas for the edge modes localized curling of the magnetization leads to a departure from the macrospin description which breaks the rotational symmetry of the system and gives an additional degree of freedom [29].

\section{A. Bulk modes}

Focusing on the $8-\mathrm{GHz}$ bulk mode (Fig. 2), changes in configuration can lead to the splitting of modes as well as shifts in the peak center of more than $1 \mathrm{GHz}$. The maximum shift is thus comparable to previously reported shifts [14,15] in reconfigurable magnonic crystals, though honeycomb ASI offers the additional benefit of a large number of intermediate configurations that allow the peak position to be smoothly tuned from one position to another. A general downward trend in the position of peak centers as the monopole number increases may be explained by the reduced effective field, $\mathbf{H}_{\mathrm{eff}}$, felt by islands as the opposing stray field from neighboring island increases.

In addition to shifting and splitting modes, changing the magnetic microstate allows the amplitude of the modes to be enhanced or suppressed (Fig. 3) suggesting applications as microwave filters or "ON/OFF" elements in magnonic circuits. Such "ON" and "OFF" microstate pairs exist in both the zero- and two-monopole states and allow enhancements by a factor of up to 30 without any net change in the total system energy, comparable to recent results in a one-dimensional reconfigurable magnonic device [30].

That it is possible to distinguish between states with the same monopole density is testament to the exquisite sensitivity of the FMR response to the internal field distribution. The distinct spectra observed for the five different monopole-free microstates may be explained in terms of the vector sum of the stray fields felt by each island from its four nearest neighbors.

For each vertex obeying the ice rule constraint (twoin/one-out or one-in/two-out), there are two islands the magnetization of which is oriented in the majority-spin direction (i.e., "in" for a two-in/one-out vertex) and one with magnetization in the minority-spin direction ("out"). Whether an island is oriented in the majority or minority direction for a particular vertex determines the stray field it feels from the other two islands at the vertex: Minority islands experience a stray field parallel ("=") to their magnetization direction whereas the stray field for majority islands is in one of the directions perpendicular ( "个" or " $\downarrow$ ") to their magnetization (see Supplemental Material [31]). Since each island subtends two vertices this means that each island experiences one of four possible combinations of perpendicular and parallel fields, which in turn may combine in one of five ways to to form a monopole-free microstate on the unit cell studied here. The combinations of different fields felt by the six islands determine the fine structure of the five zero-monopole spectra in Fig. 2-in particular, the bulk mode may be resolved into two or three submodes where the number of submodes depends on the number of distinct fields felt by the six islands.

Such differences between microstates with the same monopole density indicate that even in the part of the island where interisland interactions are sufficiently weak that the macrospin approximation holds they are nevertheless involved in modifying the high-frequency response. The role of the stray fields from neighbors is further supported by the fact that the enhancement/suppression factor between two states could be made larger by increasing the saturation magnetization, $M_{\mathrm{S}}$, so that interisland interactions were stronger. It was possible to increase the enhancement/suppression ratio by a further factor of 4 by increasing $M_{\mathrm{S}}$ to $1200 \mathrm{kA} / \mathrm{m}$. Beyond this the simulated islands were no longer stable as single ferromagnetic domains, but exploring ferromagnetic materials with high saturation magnetizations such as cobalt and tuning the gap between neighboring islands is a viable route to maximizing the enhancement/suppression ratio.

\section{B. Edge modes}

For the two modes at around 3 and $6 \mathrm{GHz}$, which are localized at the edges of islands, there is an additional degree of freedom in the way that the magnetization of the nanoislands curls up at vertices in order to minimize the magnetostatic self-interaction and interactions with neighboring islands. In this case the macrospin approximation is no longer strictly valid [16,29] and two configurations which map onto one another as macrospins may have different spectra if their island edges curve differently at vertices.

At each vertex three edges come together which may either curve (C) or remain straight as for individual islands (S), leading to four possible vertex types: SSS, SSC, CCS, and CCC (see Supplemental Material [32]). The vertex types that include curved edges may then be further characterized by whether or not all their edges curve in the same direction and if so by the sense of their chirality. This gives 11 vertex types in total and in principle $11^{4}$ different vertex configurations on 
the periodic plaquette used in this paper, offering substantial scope for tuning the high-frequency response of honeycomb ASI by controlling the edge curling.

Not all of these vertex types are stable in the absence of an external field, and, in simulations carried out at remanence, monopoles were only seen in the two chiral CCC states, while two-in/one-out and one-in/two-out vertices were only observed in achiral CCS states with the two islands with the majority-spin direction showing curling in opposite directions. Unstable vertex types may nevertheless be observed in ASI systems if they are stabilized using an external magnetic field [29].

The precise extent to which the magnetization curls at island edges depends on the competition between magnetostatic interisland and self-interactions. These interactions may be tuned via the interisland separation and the island thickness, respectively, with the edge curling vanishing when simulations were repeated with the island thickness set below $3 \mathrm{~nm}$ or the edge-to-edge separation between islands increased beyond $50 \mathrm{~nm}$ (i.e., where the dipolar field from neighboring edges becomes small compared to the magnetization of permalloy).

To determine whether the edge-localized modes are sensitive to this edge curling the four-monopole microstate was prepared with two different magnetization profiles: One with only "left-handed" vertices and one in which right- and lefthanded vertices alternate [Fig. 4(a)]. The lower frequency edge mode was found to indeed be sensitive to edge curling, while the 6-GHz edge mode was not observed to vary significantly as a result of the different magnetization profiles, and the $8-\mathrm{GHz}$ bulk mode was insensitive to edge curling as expected [Fig. 4(b)].

That the higher-frequency edge modes did not respond to the different edge curling profiles as much as the $3-\mathrm{GHz}$ modes is unexpected as their spatial profiles show a similar chiral distortion to that seen for the 3-GHz mode [Fig. 4(a)], but it is likely to be because less of the power associated with the higher-frequency edge modes is localized right along the island boundaries where curling is strongest [Fig. 1(a)].

The 3-GHz edge mode was substantially altered by moving between the different edge curling profiles. It is notable that the "mixed" profile spectrum is not simply a linear combination of the right- and left-handed profiles, attesting to the importance of edge curling in interisland interactions and in accessing the full range of high-frequency behaviors available in ASI systems [33]. We note that, in addition to an intrinsic effect on the spectrum from the magnetic configuration, real ASI systems will display additional extrinsic differences in the spectra of states with varying edge curling profiles due to roughness introduced in the fabrication that breaks symmetries. The edge curling thus creates distinct FMR fingerprints which may form the basis of a readout method with which to characterize the chirality of monopoles in ASI systems.

\section{Effect of an external magnetic field}

It was found that at remanence monopoles are only stable in chiral CCC states while unexcited vertices are only stable in an achiral CCS state, limiting the edge curling profiles available for controlling high-frequency dynamics. However, (a)
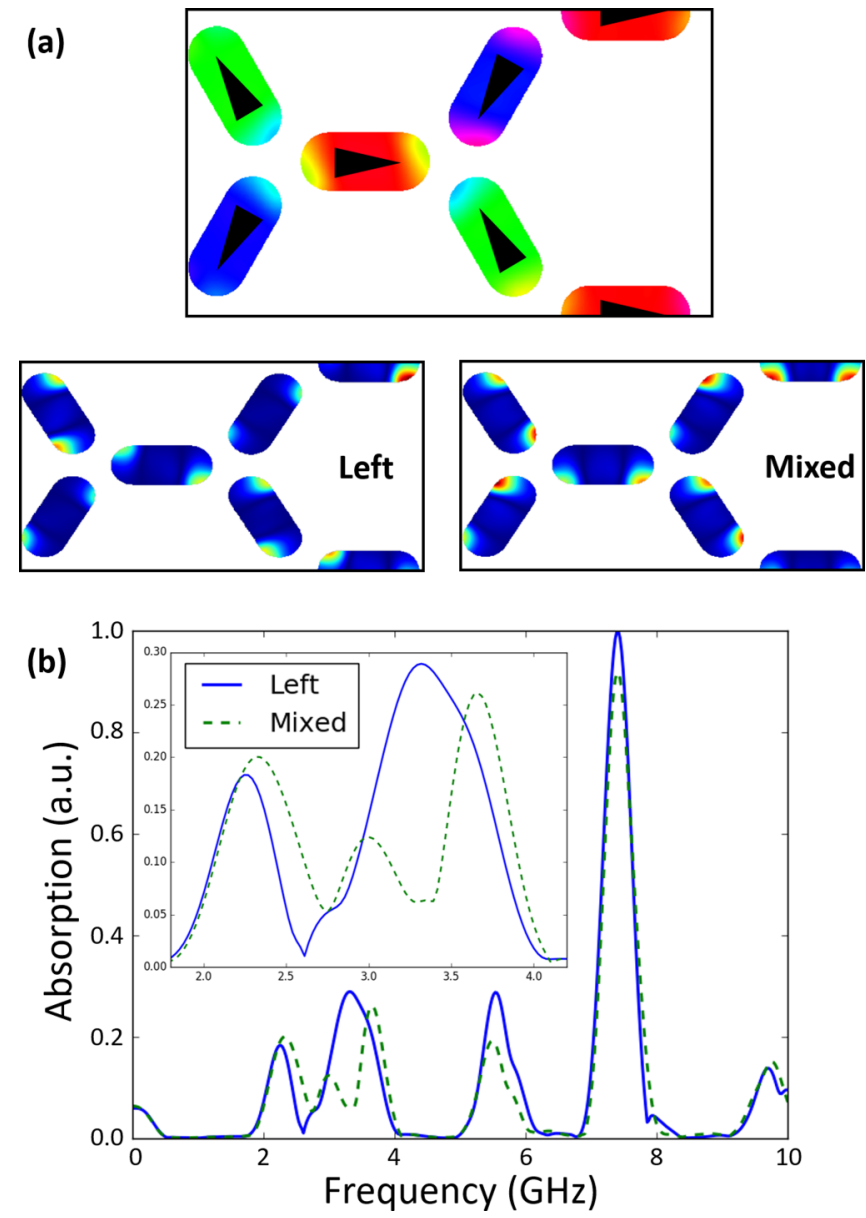

FIG. 4. (a) The four-monopole microstate (top left) was prepared with two different edge curling profiles, the effect of which can be seen in the distorted spatial profile of the $3-\mathrm{GHz}$ mode (top right, bottom). (b) Eigenmode spectra for the four-monopole microstate with the left-handed and mixed edge curling profiles. The $3-\mathrm{GHz}$ mode (inset) is particularly affected by moving between the different profiles.

the remaining vertex states may still be accessed if they are stabilized by an external magnetic field, with the additional consequence that the external field will break the rotational and time-reversal symmetries and allow configurations that map onto each other to be distinguished from each other spectrally.

The effect of an external magnetic field (applied parallel to the long axis of the honeycomb cell) on the eigenmode spectra of an ASI system with a reduced lattice parameter was simulated for a polarized state and the four-monopole state to demonstrate how the field and microstate interact to determine the high-frequency response. The spectra for these systems under a range of external fields from -100 to $+100 \mathrm{mT}$ are shown in Fig. 5, where for each field the microstate was reinitialized and allowed to relax so that the spectra are indicative of the expected response if the system is initialized and immediately subjected to a given field with no further field history.

The evolution of individual modes of the polarized configuration is generally well described by the Kittel formula for 


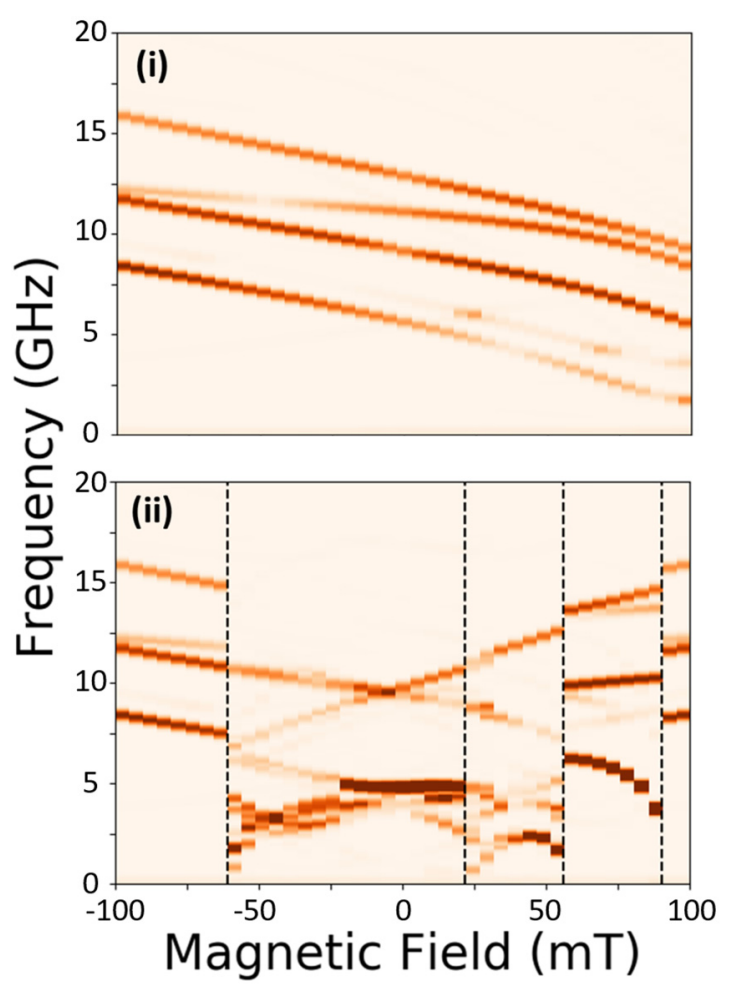

FIG. 5. Eigenmode spectra for systems initialized in the (i) polarized and (ii) four-monopole configurations [shown in Figs. 6(a) and 6(b), respectively] as a function of an externally applied field. The spectrum initialized in the four-monopole state shows the same five regimes as observed in Fig. 6.

ferromagnetic resonance:

$$
f(H) \propto \sqrt{\left(M_{\mathrm{s}}+H_{\mathrm{k}}\right)\left(M_{\mathrm{s}}+H_{\mathrm{k}}+H\right)}
$$

where $M_{\mathrm{s}}$ denotes the saturation magnetization, $H_{\mathrm{k}}$ is the effective field due to magnetic anisotropy, and $H$ is the externally applied field at which resonance occurs. Since the effective anisotropy field is increased by reducing the lateral dimensions of each island, the frequencies of the modes present at zero field in Fig. 5 are shifted upwards with respect to their positions in Fig. 1. The degeneracy between the modes of the diagonal and horizontal islands is further broken due to the broken rotational symmetry, leading to a fully separate bulk mode for the diagonal islands in which $H_{\mathrm{k}}$ does not align with the external field $H$. Rotational symmetry breaking may thus be used to introduce additional eigenmodes.

For the four-monopole state, the evolution of the eigenmode spectrum in response to an applied field is more complex as the edge curling and eventually full magnetization of islands reverse to align with the applied field (Fig. 6). At around $30 \mathrm{mT}$ in the positive $x$ direction, achiral monopoles form and are stable for as long as the field is applied (Fig. 6). As was observed at remanence only the lowest-frequency edge mode, which is here redshifted by over $1 \mathrm{GHz}$, is sensitive to a change in the edge curling profile (Fig. 5). At just above $50 \mathrm{mT}$, two diagonal islands irreversibly switch their magnetization direction and a small jump in the total magnetization triggers a remarkable change in the eigenmode spectrum, with the middle mode shifting by almost $3 \mathrm{GHz}$.
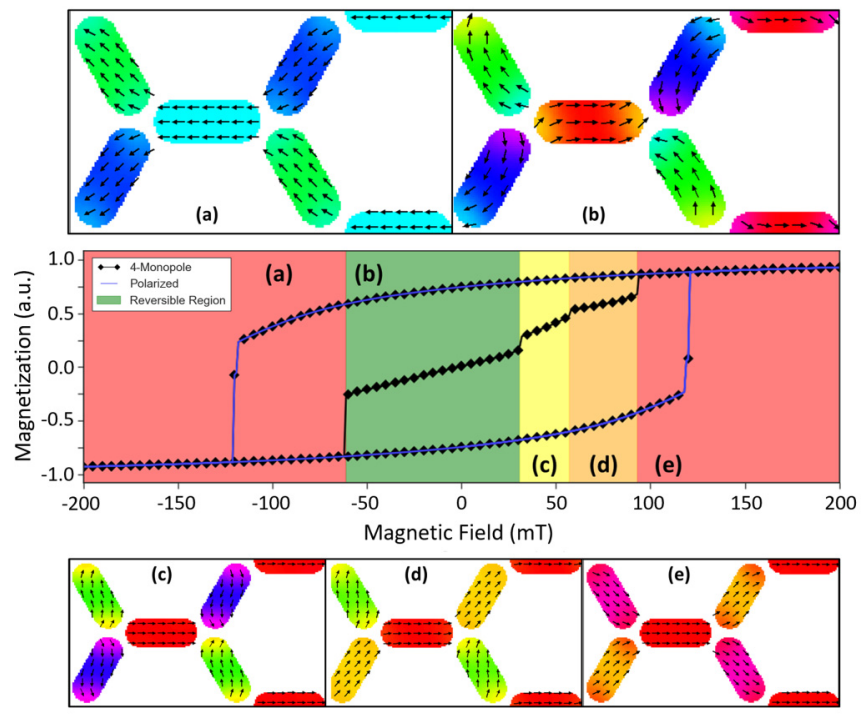

FIG. 6. Magnetization curves for a honeycomb ASI system initialized at zero field in a polarized and four-monopole configuration and allowed to relax in a magnetic field applied along the long axis of the unit cell. The field intervals over which changes in the magnetization of both configurations are reversible are shaded, showing five clear regimes: (a) left-polarized; (b) initial four-monopole state (with a mixed edge curling profile); (c) four-monopole state (with field-stabilized achiral monopoles); (d) ice-rule state; and (e) right-polarized. In regions (a) and (e) the two curves coincide since the four-monopole state is fully saturated.

The system saturates at $90 \mathrm{mT}$ (or $60 \mathrm{mT}$ applied in the negative $x$ direction) at which point its mode spectrum reproduces that of the polarized system.

The application of external fields thus offers an additional means of controlling the high-frequency response of ASI systems that complements microstate selection. Since fieldstabilized vertex curling profiles will relax back into their initial states when the external field is removed, it is possible to achieve discrete shifts in the high-frequency response without leaving the reversible region beyond which the microstate is lost. The microstate may therefore be selected to broadly determine the high-frequency characteristics using an external field applied to produce additional modes by breaking symmetry. External fields may further modulate this response either in a continuous fashion [following Eq. (2)] or in reversible discrete jumps mediated by changes in the vertex curling profile.

\section{CONCLUSION}

In summary, the high-frequency response of honeycomb ASI in different magnetic configurations has been characterized via micromagnetic simulations. For modes localized in the bulk of ferromagnetic islands, the dependence on the magnetic microstate of the system is well described within a macrospin approximation. A consequence of this is that the number of microstates with distinguishable spectra at remanence is lower than the number of microstates itself, since the eigenmode spectra are invariant under rotations and global spin reversals. This redundancy in the microstates 
activating a particular spectrum offers increased flexibility in the control of high-frequency dynamics, allowing the most convenient microstate to be selected from a range of isospectral alternatives where microstate selection is constrained. The precise degree of redundancy may be altered by exploring ASI systems with broken rotational symmetry [34-36].

Within the distinguishable microstate space significant control of the spin-wave spectrum was achieved by moving between different microstates. The bulk mode may be shifted by as much as $1 \mathrm{GHz}$, split into discrete modes or switched off by moving between the ten nonredundant microstates available on the six-island plaquette studied here. Stronger mode enhancement/suppression is achievable in systems where the interisland interaction strength is increased. Increasing the number of nanoislands introduces further degrees of configurational freedom, offering even greater scope for sculpting the dynamic response which scales extensively with system size. The large number of near-degenerate configurations in ASI systems mean that the spin-wave spectrum may be smoothly tuned-in particular increasing the monopole defect density gradually lowers the frequency of the bulk mode as opposing magnetostatic fields reduce the effective field felt by the system, with the "resolution" of tuning improving with system size. This demonstrates the benefits of large, fully accessible microstate space and overcomes a key limitation of previously explored reconfigurable magnonic crystal concepts [14,15], which support only a limited number of stable configurations.

Modes localized at island edges were found to be sensitive to curling in the magnetization neglected in the macrospin picture. This edge curling provides an additional degree of freedom with which to control the high-frequency response, with the application of external fields stabilizing different types of vertex curling. External fields also break rotational and time-reversal symmetry, introducing additional modes and allowing further tuning of high-frequency dynamics by breaking degeneracies resulting from the symmetries of a macrospin system.

A combination of global external fields and local nanoisland stray fields determined by the ASI microstate thus allows substantial control over the high-frequency response of ASI systems. The significance of this is heightened by recently developed techniques for state control [37] and island-specific magnetic writing [38-40], bringing fine spectral control within experimental reach by providing a method of reliably moving between microstates. The macroscopic degeneracy of ASI systems ensures a colossal range of stable configurations the varying properties of which may be selected to precision tune the response of prospective devices ranging from programmable microwave filters to reconfigurable magnonic components. The lattice symmetry and symmetry-breaking effects of interisland interactions provide a broad range of parameters for the rational design of versatile, highly tunable spectrum-selection devices inviting a host of applications from microwave filters to reconfigurable functional magnonic elements for low-power data processing.

\section{ACKNOWLEDGMENTS}

W.R.B. was supported by the Engineering and Physical Sciences Research Council (Grant No. EP/G004765/1) and the Leverhulme Trust (Grant No. RPG 2012-692). The authors would further like to thank Prof. L. F. Cohen for illuminating discussions and the Imperial College Research Computing Service for access to their high-performance computing facilities.
[1] R. F. Wang, C. Nisoli, R. S. Freitas, J. Li, W. McConville, B. J. Cooley, M. S. Lund, N. Samarth, C. Leighton, V. H. Crespi, and P. Schiffer, Artificial 'spin ice' in a geometrically frustrated lattice of nanoscale ferromagnetic islands, Nature (London) 439, 303 (2006).

[2] C. Nisoli, R. Moessner, and P. Schiffer, Colloquium: Artificial spin ice: Designing and imaging magnetic frustration, Rev. Mod. Phys. 85, 1473 (2013).

[3] L. J. Heyderman and R. L. Stamps, Artificial ferroic systems: Novel functionality from structure, interactions and dynamics, J. Phys.: Condens. Matter 25, 363201 (2013).

[4] S. Ladak, D. E. Read, G. K. Perkins, L. F. Cohen, and W. R. Branford, Direct observation of magnetic monopole defects in an artificial spin-ice system, Nat. Phys. 6, 359 (2010).

[5] E. Mengotti, L. J. Heyderman, A. Fraile Rodriguez, F. Nolting, R. V. Hügli, and H. B. Braun, Real-space observation of emergent magnetic monopoles and associated Dirac strings in artificial kagome spin ice, Nat. Phys. 7, 68 (2011).

[6] J. P. Morgan, A. Stein, S. Langridge and C. H. Marrows, Thermal ground-state ordering and elementary excitations in artificial magnetic square ice, Nat. Phys. 7, 75 (2010).

[7] It should be noted that in some previous studies the term "monopole" has been used to describe excitations with respect to a reference background state, so that in honeycomb ASI a two-in/one-out vertex would be counted as a monopole if the same vertex had a one-in/two-out in the reference state. In this paper, we reserve the term "monopole" for vertices with an all-in or all-out configuration, so that they act as a convenient way to characterize the total energy of the system with no need to specify a reference state.

[8] S. Gliga, A. Kákay, R. Hertel, and O. G. Heinonen, Spectral Analysis of Topological Defects in an Artificial Spin-Ice Lattice, Phys. Rev. Lett. 110, 117205 (2013).

[9] V. S. Bhat, F. Heimbach, I. Stasinopoulos, and D. Grundler, Magnetization dynamics of topological defects and the spin solid in a kagome artificial spin ice, Phys. Rev. B 93, 140401(R) (2016).

[10] M. Krawczyk and D. Grundler, Review and prospects of magnonic crystals and devices with reprogrammable band structure, J. Phys.: Condens. Matter 26, 123202 (2014).

[11] A. V. Chumak, V. I. Vasyuchka, A. A. Serga and B. Hillebrands, Magnon spintronics, Nat. Phys. 11, 453 (2015).

[12] A. V. Chumak, A. A. Serga, and B. Hillebrands, Magnonic crystals for data processing, J. Phys. D 50, 244001 (2017).

[13] E. Iacocca, S. Gliga, R. L. Stamps, and O. G. Heinonen, Reconfigurable wave band structure of an artificial square ice, Phys. Rev. B 93, 134420 (2016). 
[14] J. Topp, D. Heitmann, M. P. Kostylev, and D. Grundler, Making a Reconfigurable Artificial Crystal by Ordering Bistable Magnetic Nanowires, Phys. Rev. Lett. 104, 207205 (2010).

[15] R. Verba, G. Melkov, V. Tiberkevich, and A. N. Slavin, Fast switching of a ground state of a reconfigurable array of magnetic nano-dots, Appl. Phys. Lett. 100, 192412 (2012).

[16] S. Gliga, A. Kákay, L. J. Heyderman, R. Hertel, and O. G. Heinonen, Broken vertex symmetry and finite zero-point entropy in the artificial square ice ground state, Phys. Rev. B 92, 060413(R) (2015).

[17] M. B. Jungfleisch, W. Zhang, E. Iacocca, J. Sklenar, J. Ding, W. Jiang, S. Zhang, J. E. Pearson, V. Novosad, J. B. Ketterson, O. G. Heinonen, and A. Hoffmann, Dynamic response of an artificial square spin ice, Phys. Rev. B 93, 100401(R) (2016).

[18] V. S. Bhat, F. Heimbach, I. Stasinopoulos, and D. Grundler, Angular-dependent magnetization dynamics of kagome artificial spin ice incorporating topological defects, Phys. Rev. B 96, 014426 (2017).

[19] X. Zhou, G.-L. Chua, N. Singh, and A. O. Adeyeye, Large area artificial spin ice and anti-spin ice $\mathrm{Ni}_{80} \mathrm{Fe}_{20}$ structures: Static and dynamic behavior, Adv. Funct. Mater. 26, 1437 (2016).

[20] M. B. Jungfleisch, J. Sklenar, J. Ding, J. Park, J. E. Pearson, V. Novosad, P. Schiffer, and A. Hoffmann, High-Frequency Dynamics Modulated by Collective Magnetization Reversal in Artificial Spin Ice, Phys. Rev. Appl. 8, 064026 (2017).

[21] V. S. Bhat and D. Grundler, Angle-dependent magnetization dynamics with mirror-symmetric excitations in artificial quasicrystalline nanomagnet lattices, Phys. Rev. B 98, 174408 (2018).

[22] I. Gilbert, G.-W. Chern, B. Fore, Y. Lao, S. Zhang, C. Nisoli, and P. Schiffer, Direct visualization of memory effects in artificial spin ice, Phys. Rev. B 92, 104417 (2015).

[23] F. Montoncello, L. Giovannini, W. Bang, J. B. Ketterson, M. B. Jungfleisch, A. Hoffmann, B. W. Farmer, and L. E. De Long, Mutual influence between macrospin reversal order and spinwave dynamics in isolated artificial spin-ice vertices, Phys. Rev. B 97, 014421 (2018).

[24] W. Bang, F. Montoncello, M. B. Jungfleisch, A. Hoffmann, L. Giovannini, and J. B. Ketterson, Angular-dependent spin dynamics of a triad of permalloy macrospins, Phys. Rev. B 99, 014415 (2019).

[25] Simulations were carried out on high-performance computing facilities maintained by the Imperial College Research Computing Service, doi: 10.14469/hpc/2232.

[26] A. Vansteenkiste, J. Leliaert, M. Dvornik, M. Helsen, F. GarciaSanchez, and B. Van Waeyenberge, The design and verification of MuMax3, AIP Adv. 4, 107133 (2014).

[27] M. Dvornik, Y. Au, and V. V. Kruglyak, Micromagnetic simulations in magnonics, in Magnonics: From Fundamentals to
Applications, edited by S. O. Demokritov and A. N. Slavin (Springer, New York, 2013), pp. 101-115.

[28] A. Baker, M. Beg, G. Ashton, M. Albert, D. Chernyshenko, W. Wang, S. Zhang, M.-A. Bisotti, M. Franchin, C.-L. Hu, R. L. Stamps, T. Hesjedal, and H. Fangohr, Proposal of a micromagnetic standard problem for ferromagnetic resonance simulations, J. Magn. Magn. Mater. 421, 428 (2017).

[29] N. Rougemaille, F. Montaigne, B. Canals, M. Hehn, H. Riahi, D. Lacour, and J.-C. Toussaint, Chiral nature of magnetic monopoles in artificial spin ice, New J. Phys. 15, 035026 (2013).

[30] A. Haldar, D. Kumar, and A. O. Adeyeye, A reconfigurable waveguide for energy-efficient transmission and local manipulation of information in a nanomagnetic device, Nat. Nanotechnol. 11, 437 (2016).

[31] See Supplemental Material at http://link.aps.org/supplemental/ 10.1103/PhysRevB.100.214425 for further discussion on the different ways in which the stray fields from nearest neighbors may combine.

[32] See Supplemental Material at http://link.aps.org/supplemental/ 10.1103/PhysRevB.100.214425 for a more detailed discussion of the different types of edge curling.

[33] Y. Li, G. Gubbiotti, F. Casoli, F. J. T. Gonçalves, S. A. Morley, M. C. Rosamond, E. H. Linfield, C. H. Marrows, S. McVitie, and R. L. Stamps, Brillouin light scattering study of magneticelement normal modes in a square artificial spin ice geometry, J. Phys. D 50, 015003 (2017).

[34] M. J. Morrison, T. R. Nelson, and C. Nisoli, Unhappy vertices in artificial spin ice: New degeneracies from vertex frustration, New J. Phys. 15, 045009 (2013).

[35] G.-W. Chern and P. Mellado, Magnetic monopole polarons in artificial spin ices, Europhys. Lett. 114, 37004 (2016).

[36] T. Dion, D. M. Arroo, K. Yamanoi, T. Kimura, J. C. Gartside, L. F. Cohen, H. Kurebayashi, and W. R. Branford, Tunable magnetization dynamics in artificial spin ice via shape anisotropy modification, Phys. Rev. B 100, 054433 (2019).

[37] D. M. Burn, M. Chadha, and W. R. Branford, Dynamic dependence to domain wall propagation through artificial spin ice, Phys. Rev. B 95, 104417 (2017).

[38] J. C. Gartside, D. M. Arroo, D. M. Burn, V. L. Bemmer, A. Moskalenko, L. F. Cohen, and W. R. Branford, Realization of ground state in artificial kagome spin ice via topological defectdriven magnetic writing, Nat. Nanotechnol. 13, 53 (2018).

[39] Y.-L. Wang, Z.-L. Xiao, A. Snezhko, J. Xu, L. E. Ocola, R. Divan, J. E. Pearson, G. W. Crabtree, and W.-K. Kwok, Rewritable artificial magnetic charge ice, Science 352, 962 (2016).

[40] M. Pancaldi, N. Leo and P. Vavassori, Selective and fast plasmon-assisted photo-heating of nanomagnets, Nanoscale 11, 7656 (2019). 